\title{
Using Mobile Applications to Reinforce Language Concepts for Children with Autism:Case Study
}

\section{Dr /Aml Gameel Aly El-morsy ${ }^{1}$}

\begin{abstract}
Recent years have witnessed major advances in technologies to support children diagnosed with Autism. Since autistic children suffer from learning disabilities, language lack and communication barrier. A number of Mobile applications have impacted their daily lives, with the goal of enhancing their language and abilities to interact and communicate with others. Hence, the current study was conducted to examine the impact of mobile apps to reinforce language concepts for autistic children. The study focused on the usability of Arabic mobile app (TAC). The sample included autistic children who are minimally effective communicators. The participants of the current study were two children with autism enrolled in one of Autism Center in Hurdada, Egypt, aged between 5-6 years almost. The results showed an encouraging impact of the mobile app (TAC) in supporting autistic children to enhance language concepts.
\end{abstract}

Keyword: Mobile App (Mobile Application), Language Concepts, Autism

${ }^{1}$ Department of Kindergarten, Education College, King Khalid University, Abha, Saudi Arabia 


\section{مستخلص البحث}

دراسة بعنوان استخدام تطبيقات الجوال لتعزيز المفاهيم اللغوية لدى الأطفال المصابين بالتوحد، حيث هدفت الدراسة إلى التعرف على مدى فاعلية تطبيقات الجوال لتعزيز المفاهيم اللغوية لدى الأطفال المصابين بالتوحد، وقد أجريت الدراسة على عينة تكونت من (r) الأطفال بمرحلة رياض الاطفال، و تتراوح اعمارهم ما بين (0-7) سنوات تقريبا من للأطفال المصابين بالتوحد، وقد أختيرت عينة البحث بطريقة عمدية من الأطفال ما قبل المدرسة بحيث حققت الشروط الواجبة للعينة، وطبقت الدراسة المنهج شبه التجريبى، ومجموعة تجريبية واحدة، واستمرت التجربة لمدة ستة أسابيع. وأظهرت النتائج مدى فعالية تطبيقات الجوال لتعزيز المفاهيم اللغوية لدى الأطفال المصابين بالتوحد.

الكلمات المفتاحية : نطبيقات الجوال، المفاهيم اللغوية، الأطفال المصابين بالتوحد 


\section{Introduction}

Nowadays, mobile technologies are becoming increasingly ubiquitous and networked. Such technologies can be used creatively in different areas. Using mobile technologies in education is a clear example of such innovation. Mobile devices equipped with internet connections have created the need for a new form of electronic learning, called mobile learning ( $\mathrm{Fu}, \mathrm{Su}$, and $\mathrm{Yu}$, 2009). Internet-enabled mobile devices can help children to access learning resources and online courses, anywhere and at any time. Technology has the potential to support all children. There are obvious cases where technologies are created to support children with autism (Bausch, Ault \& Hasselbring, 2015).

However, in many cases technology must be retrofitted to meet the diverse needs of all children with autism (SanchezGordon \& Luján-Mora, 2016). The same is true with mobile devices and applications. The arrival of mobile technology in the mainstream has led to changes not only in society at large but also in evaluation and treatment of individuals with communication disorders (McNaughton \& Light 
2013). In particular, mobile technologies have become extremely popular tools for individuals with autism.

Socially, children with autism can be seen as being quite 'awkward' due to their significant impairments in nonverbal behaviors, such as eye contact and understanding facial expressions. Children with autism lack the spontaneous seeking of others to share enjoyment or achievements with, for instance, joint attention (Paparella et al, 2011). Due to these impaired social interactions children with autism tend to play solitary activities and avoid participation in social games, especially with peers. Severe language impairments are a common characteristic of autism (DSM-V, 2013). Approximately 80\% of children with autism aged 5 years and younger who enter special education are non-verbal (Anderson et al., 2007). These linguistic difficulties can have a devastating impact on children's capacity to communicate, but special educators have developed numerous interventions that enable communication without expressive language.

However, the emergence of the mobile phone has elicited a surge toward technology-mediated education and 
interventions, which may benefit children with autism due to the device's portability, easy to use touchscreen interface, and ability to emit multimodal output (Lofland, 2016). While there are countless software applications "apps" designed to support language development and communication using digital pictures, little research has investigated the ability of children with autism to comprehend and learn from symbolic information presented via mobile applications.

Similarly, there is little guidance as to how the features of electronic apps can be maximized to specifically facilitate learning for children with autism. These are of inquiry are, however, rapidly emerging. Mobile applications (apps) are increasingly being used with children with autism to enhance some language concepts for autistic children. The use of mobile devices for learning could expand the environment or scope of learning at all times and at all places. Modern developments in mobile applications for children with autism defective children using some of the existing mobile applications have shown that mobile information and communication technologies could 
encourage and boost some language concepts for autistic children.

The awareness of mobile applications evokes a series of responses in children with autism towards their learning language concepts. It is important for autistic children to have a good understanding of different concepts as it assists in improving some language concepts for children with autism. In order for a child to use concepts in their spoken language, they need to have a good understanding of what these concepts are and what they mean. By developing this understanding they are then better able to follow instructions at home, at preschool, and in the school environment.

\subsection{The context of the Problem}

The current study addressed the question of whether or not autistic children benefit from mobile apps in development of some language concepts. The problem of the current study was derived from a personal observation of the researcher that showed the autistic children have difficulty in discriminating some language concepts. They have 
weaknesses in language skills and this affects their ability to decode with efficiency.

\subsection{Statement of the problem}

Autistic children often have language concepts problem. So, the researcher suggested the application of the mobile apps to solve the target autistic children's enhancement some language concepts problem.

\subsection{Study Significance}

The current study was thought to be important for the following:

a) Enhancing autistic children's language concepts.

b) Proposing suitable instructional applications to aid the autistic children in enhancing language concepts.

\subsection{Study Aims}

It aimed at the following:

a. Identifying language concepts needed for the autistic children.

b.Identifying the effect of the mobile apps on enhancing autistic children's language concepts.

\subsection{Study Questions}


The current study attempted to provide answers to the following questions:

a)What are the language concepts necessary for autistic children?

b) What is the effect of using mobile applications in enhancement some language concepts for children with autism?

\subsection{Study Hypotheses}

To address the following hypotheses are constructed:

1. There are statistically significant differences between the mean ranks of the children on the the pre/post mobile application in favor of the post application.

2. There are no statistically significant differences between the mean ranks of the children in the post application and the follow-up of the mobile application.

\subsection{Study Delimitations}

This study was delimited to the following:

a. Some language concepts: vocabulary development (Indicates an object when given name \& Names of objects) b. A sample of autistic children in kindergarten.

\subsection{Definition of Terms}


1.8.1. Mobile applications (also known as mobile apps) are software programs developed for mobile devices such as smartphones and tablets which are created for different purposes.

1.8.2. Language concepts are the foundation of a child's education. They are words that a child needs to understand in order to perform everyday tasks like following directions, participating in classroom routines, and engaging in conversation.

1.8.3. Autism is a neurodevelopmental disorder that affects the development of the brain during the first 3 years of a child's life. There are three main areas of difficulty that people with autism experience, otherwise known as the triad of impairments. These include impaired communication, impaired social skills and restricted repetitive and stereotyped patterns of behaviors, interests, and activities as stated in DSM (DSM-V, 2013).

\section{Review of Literature}

\subsection{Mobile Apps}

Mobile apps can help autistic children and their social environment particularly in the sphere of education and 
communication. Thanks to the personal adaptation and use of specific technology in their daily lives, we are observing that children with autism can be helped to relate better to others, to learn, and to feel better in themselves. The advent of mobile applications has opened up the field of learning via technology, and the use of electronic applications on these devices continues to dramatically rise for children with communication and social impairment, specifically those with autism (Hartley \&Allen, 2015).

\subsubsection{Importance of Mobile Apps for Autistic Children}

In light of the ever-growing number of "autism apps", it is increasingly important that help autistic children to communicate in different environments and places. So, mobile apps lead to enhance some language concepts of children with autism, their portability also enables autistic children to perform learning activities anytime and anywhere. Apps are developed to allow fine-grained customization of pictorial features, which could usefully support learning in autism. It is easier to program an app than to print a book to present multiple differently-colored examples of a target referent rather than a single exemplar. 
Such features could be used when teaching language concepts to highlight the similarity of shape, and foster shape-based generalizations in autistic children. Children with autism frequently generalized to items that matched depicted objects on shape or color. Thus, it appears that minimally-verbal autistic children do not know intuitively what names refer to when paired with pictures (i.e., the picture itself, the depicted object's shape, or the depicted object's color) and their symbolic comprehension is significantly influenced by the type of picture. Taken together, these differences suggest that there might be an atypical route of word learning via pictures in autism (Hartley \&Allen, 2014).

\subsubsection{The Benefits of Mobile Apps for Autistic Children}

An impressive list of apps designed for children with autism. The use of mobile apps aims at helping autistic children enhance some language concepts using mobile devices. Most autistic children are visual learners and mobile technology appeals to them. They embrace mobile technology and enjoy using smartphones and tablets to learn and play and a lot of apps have been developed for them 
(Urturi, et al, 2011). Apps enable the creation and integration of customized visual supports. Personalized stimuli of this nature improve the specificity of children's communication and expand opportunities for interaction (Hartley \&Allen, 2015). Thus, when used mobile apps can effectively promote language concepts and communication in minimally-verbal children with autism.

2.2. Autism is a disorder of social development, language, and communication. Autistic children have inappropriate behaviors and attention. Autism varies in symptoms. One of many characteristics is that they isolate themselves and show less attention to their environments. They hardly speak or have difficulties in communication. Some do not respond or have less eye contact when they are called. They neither care for other children nor have proper plays. Since they are development impaired in personal interaction development, they do not accept stimulus from outsiders. In other words, they respond to stimulus for a short period and are often easily distracted. Severe language impairments are a common characteristic of autism (DSM-V, 2013). Approximately $80 \%$ of children with autism aged 5 years 
and younger who enter special education are non-verbal and $30 \%$ are minimally-verbal at 9-years (Anderson et al., 2007). These linguistic difficulties can have a devastating impact on children's capacity to communicate.

\subsection{Language Development of Children with Autism}

Autism, characterized by deficits in social interaction and language such as impaired conversational patterns, and abnormal and limited spoken language, is becoming one of the most prevalent disorders in children today. Deficits in language development have been widely studied, as they are often considered a defining characteristic of autism. Extant evidence has identified three common deficits in the language development of children with autism: early language delays, atypical language production, and discourse/ pragmatic difficulties (Roberts, 2014). Children with autism generally don't produce their first words until around 38 months. In addition, delays in both receptive and expressive languages have been identified, with receptive language found to be the most severely impacted (Hudry, et al., 2010). The language development of children with autism is also characterized by several atypical language 
features, such as echolalia, excessive use of jargon, and varying intonation/ prosody. Echolalia refers to the imitation and repetition of speech from others and can be classified into three types: a) Exact echolalia/imitation,
Mitigated/modified echolalia, and c) Delayed echolalia.

Research has shown that echolalia can serve as a communicative strategy for autistic children to reduce their cognitive load, to help them initiate or maintain conversations, and as a language acquisition technique. Research regarding echolalia behaviors as a specific feature of autism has indicated that they are a sign of language progression among autistic children. Specifically, the employment of echolalia strategies develops as autistic children gain linguistic competence. Most commonly, these children utilize the third type of echolalia, mitigated, to communicate by editing the words or sentences of others (Roberts, 2014).

\subsection{Language concepts for children with autism}

It is important for children to have a good understanding of different concepts as it assists in their ability to follow instructions and be specific in what they are talking about. 
In order for a child to use concepts in their spoken language, they first need to have a good understanding about what these concepts are and what they mean. By developing this understanding they are then better able to follow instructions at home, at preschool and in the school environment. Language concepts help autistic children to understand about direction, location, position, number, quantity, sequence, attributes, dimension, size and similarities and differences. The structure of language that helps autistic children to become more specific in their understanding and use of language is the knowledge of concepts. A number of mobile applications have impacted their daily lives, with the goal of enhancing their abilities to understand, interact and communicate with others. The usability of mobile applications developed for children with autism is important to enhance some language concepts. This study focuses on using mobile applications available in the Apple Store to enhance some language concepts of autistic children (Wiig, 2004). 


\section{Method}

\subsection{Sample}

The participants of the current study were four children with autism enrolled in one of Autism Center in Hurdada, Egypt, aged between 5-6 years almost. The autistic children participated from the 9:00 a.m. to 10:00 a.m., respectively. The autistic children are supposed to enhance their language concepts.

\subsection{Materials}

\section{Mobile Application Description}

The major purpose of the study is to discover whether autistic children would enhance some language concepts. For the study researched a sample of 4 autistic children, they have been asked to use mobile application: "TAC". During experimentation, the group was asked to use the mobile applications available in the Apple Store. At the end of the treatment, participants were evaluated. The App includes various activities to practice and consolidate language concepts of autistic children. Choosing these tasks based on varying in the level of difficulty. In addition, these tasks are in Arabic language; however, there is a deferent 
between two accents, which are the Modern Standard Arabic (MSA), and the classical Arabic. By identifying their areas of interest and their patterns of eye movement, we will be better informed about child preferences. This in turn will contribute to a clearer understanding of children's needs.

Also, they make communication fun, like another game on this device. Tap a picture and the application speaks. Each picture can lead to another screen of pictures. The main purpose of the app is to assist autistic children in enhancing some language concepts. This application supports only the Arabic language and emphasizes supporting verbal communication in local dialects as well as Modern Standard Arabic (MSA). The application focuses on MSA because of the dominance of using local dialects in clinical settings for speech and language therapy as well as everyday communication in non-formal settings such as at home, leisure, and in most contexts in schools and at work as shown in Figure 1. 


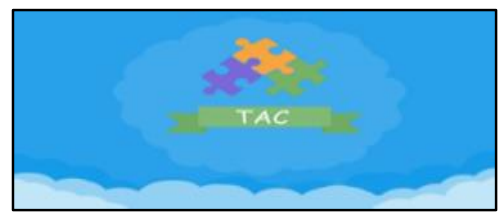

Figure1. "TAC App"

TAC application includes two tasks. The tasks that were selected for this study is as the following: "Concepts" and "Skills" in the application. The items in the application are divided into four main categories. Moreover, users can tab and choose any picture in this application. Under each picture, there is a language concept that helps the user as shown in Figure 2.

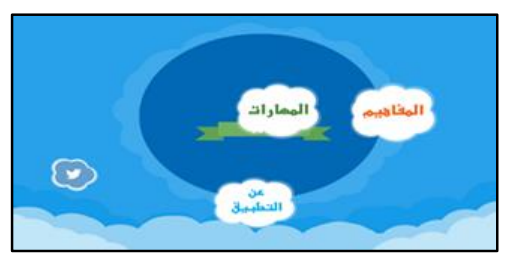

Figure 2. Main menu of "Concepts" and "Skills"

The main menu, as shown in Figure 2, provides with two major features: Main menu of Skills learning: This functionality, as shown in Figure 2.1, further divides into 4 sub-categories such as communication, self-care, new words, and grouping of the words. Each sub-category has its own pool of pictures and audio cues accordingly e.g. if a user selects shapes, the list of different shape will be shown where autistic children can tap every picture and listen to the audio cue associated with that picture.

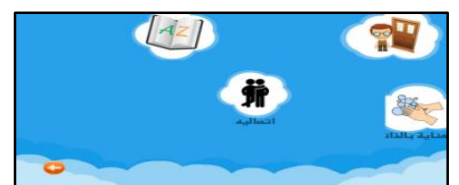

Figure 2.1. The main menu of Skills learning

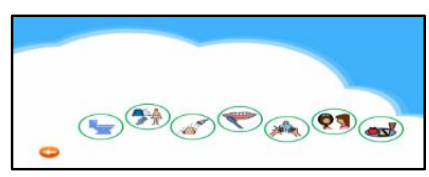

Figure 2.1.1 


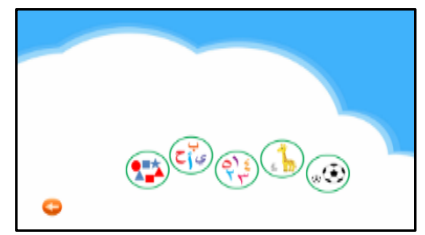

Figure 2.1.2

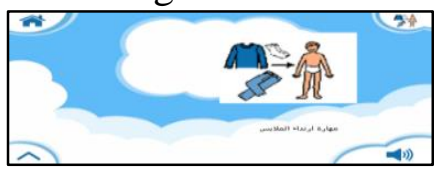

Figure 2.1.1 a

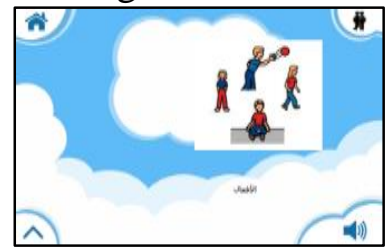

Figure 2.1.1.b

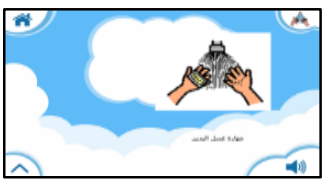

Figure 2.1.1.c

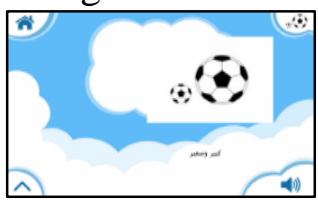

Figure 2.1.1. d

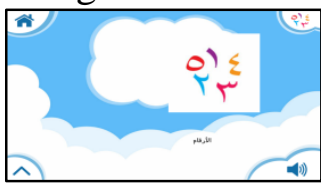

Figure 2.1.1.e

Shapes: This screen, as shown in Figure 2.1.2, pictures of different shape where autistic children can tap the picture and get to listen to the word.

Motions: As shown in Figure 2.1.1.b, this screen contains the pictures of different motions where autistic children can tap the picture and listen to the word and can learn to initiate the basic communication.

Sentences: As demonstrated in Figure 2.1.1, this functionality offers the autistic children to make complete sentences followed by the picture of the item requested. For communication, children will pick pictures from pictures array to create a sentence as shown in Figure 2.1.1.a, like; "I change my clothes" and "I wash my hands" as shown in Figure 2.1.1.c, etc. These sentences also include adjectives, verbs, and prepositions. 



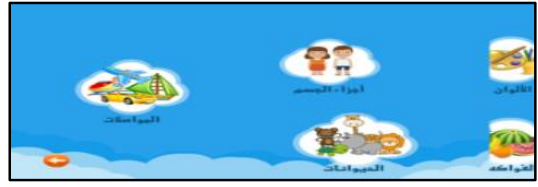

Figure 2.2.The main menu of Single-concept learning

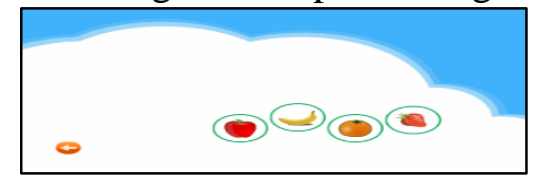

Figure 2.2.1. Fruits

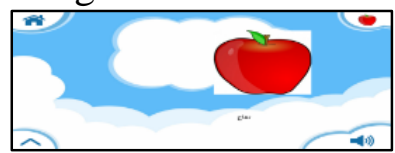

Figure 2.2.1.a

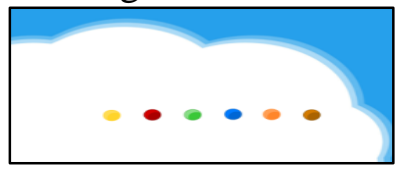

Figure 2.2.2. Colours

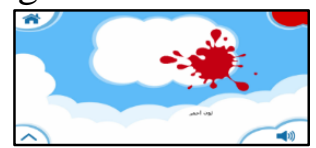

Figure 2.2.1.a

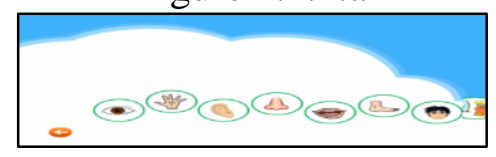

Figure 2.2.3. Parts of body

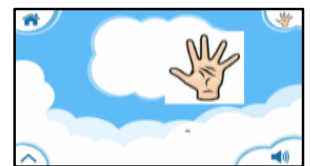

Figure 2.2.3.a

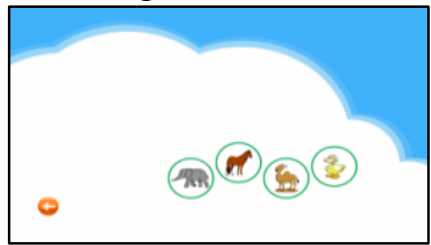

Figure 2.2.4. Animals

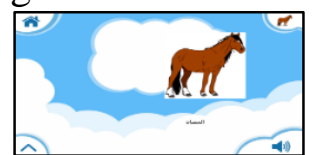

Figure 2.2.4.a

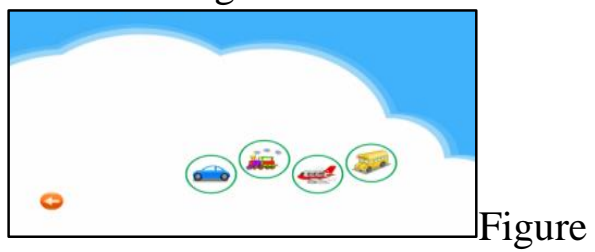

2.2.5. Transportation

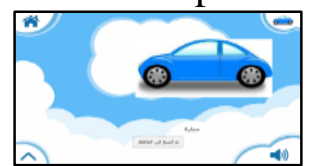

Figure 2.2.5.a

Fruits: As shown in Figure 2.2.1, the pictures of different fruits are presented so that the autistic children can tap the picture and listen to the word and can learn to initiate the basic communication.

Colors: It has pictures of different colors where the autistic children can tap the picture and get to listen to the word, as shown in Figure 2.2.2. 
Animals: The animals screen has a picture of various animals where autistic children can tap the picture and get to listen to the word, as shown in Figure 2.2.4.

Transportation: The transportation screen has pictures of various transportation where autistic children can tap a picture and listen to the word, as shown in Figure 2.2.5.

The researcher conducted the study using a mobile app (TAC) and their implication on Arabic language concepts with using different, careful, and selected techniques aiming at developing some language concepts of autistic children's ability. According to the implementation of the mobile app (TAC), all participants performed the task correctly in the application.

In the second part, we used automatic observation in the study. The application that has been used for this study is only free Arabic application available for autistic children in the App Store, and its name is "TAC".

\subsection{Research Design}

This study is conducted using a quasi-experimental research design with an independent variable for one group, autistic children who exposed to the mobile app (TAC).

\subsection{Instrumentation}


To investigate the effect of the mobile app on enhancing autistic children's language concepts.; the researcher dealt with the following instruments:

1. The mobile app (TAC) to enhance autistic children's language concepts.

2. Assessment Checklist.

\subsection{Duration of the Implementation}

Time of the implementation lasted for six weeks. Thus, all the experimentation took twenty-four sessions (twenty-four hours). The first session included the interactive and guided mobile app (TAC) activities and components of the mobile app (TAC): "Concepts and Skills" in the application. Autistic children had been involved in a variety of activities that were targeted to promote their some language concepts.

\subsection{Assessment Checklist}

This assessment, that is especially helpful in adjudging mobile app on enhancing autistic children's language concepts.

\subsection{Purpose of the assessment checklist:}

To determine the need for assessment checklist, the instrument was specifically designed to provide information to answer the study question: What is the effect of the mobile app on enhancing autistic children's language concepts? 
It gave the researcher valuable insights to adjudge autistic children's use of the mobile app.

\subsection{The content of the assessment checklist}

The assessment checklist was composed of two areas - in nine strands (Expressive Language \& Auditory Language) - purposefully designed to measure jury's level of agreement concerning the assessment checklist necessary for autistic children. High scores indicated a higher level of the agreement while low scores indicated lower levels of agreement. The overall calculated score that determined the need for assessment checklist is shown in Table 1.

Table 1. The content of the assessment checklist

This assessment, which is particularly useful in enhancing language

\begin{tabular}{|l|l|}
\hline $\begin{array}{c}\text { Language Concepts } \\
\text { Areas }\end{array}$ & \multicolumn{1}{c|}{ Language Concepts Indicators } \\
\hline \multirow{2}{*}{ Expressive Language } & $\begin{array}{l}\text { Use complete word /sentences } \\
\text { Increase vocabulary } \\
\text { Improve fluency } \\
\text { Use nouns } \\
\text { Use many multi-syllabic words }\end{array}$ \\
\hline \multirow{3}{*}{ Auditory Language } & $\begin{array}{l}\text { Understand words } \\
\text { Comprehened words } \\
\text { Recognize adjectives and colors } \\
\text { Understand complicated sentences }\end{array}$ \\
\hline
\end{tabular}

concepts, gave the researcher valuable insights to adjudging autistic children's use of the mobile app (TAC). The researcher used assessment checklist as assessment. In addition, the researcher needed 
to have a written record of observations during the children's work, which provided:

(b) A mechanism for fair and consistent feedback to the autistic children.

(b) A way of making sure that every autistic child was being observed in a timely fashion.

(b) Assurance of the information from observations was not forgotten over time.

\subsection{Validity of the assessment checklist}

In order to establish content validity for the assessment checklist, a group of jury members approved its validity and its suitability for autistic children. These experts were asked to review the items on the list and to state if given items were relevant or not. They were asked whether there were any important points that were not included in the list. They approved it and suggested modifying some components. By basing the list on best practices from the literature, construct validity was established.

\subsection{Reliability of the assessment checklist}

To determine the reliability of the assessment checklist, inter-rater reliability was used. Inter-rater reliability was calculated by finding the percentage agreement between two raters. The percentage agreement was calculated by dividing the number of times in agreement by a total number of ratings. The inter-rater reliability of the assessment checklist was (91.3\%) which is highly reliable.

\subsection{Procedures and Experimental Design}


The implementation of app introduced a variety of activities to involve the participants in individual work. At the end of the sessions included assessment and evaluation. Their teacher filled out these children's questionnaires because their literacy skills are limited. The aim of these sessions was to prepare autistic children for the intervention. The researcher has conducted the study using the mobile app. The participants of the study were trained to use mobile app (TAC) activities. The autistic children were also taught many language concepts through using mobile apps which had made enhancing language concepts activities more enjoyable. The training was expected to cause a change in their language concepts of autistic children.

Generally, autistic children were requested to use "TAC" application for 30 minutes in each session for six weeks, four sessions per week. The rules that define the method assumed in our experiments are the following:

$>$ The autistic children are considered to have learned a new word when they react while hearing the word and know how to pronounce it.

Instant pronunciation of a word and image display when an object is encountered; applications remains silent for a minute when the child touches an object's image on the smart device, after the oneminute break the object can be identified again; \& Up to $30 \mathrm{~min}$. the session with the application.

$>$ The participants were asked to accomplish two major features. 
$\checkmark$ Baseline phase refers to the status of the subject prior to intervention. Repeated measurements of the dependent variable are taken to serve as a control and enable to see the changes that occur with intervention. This phase is conducted without the use of prompts or reinforcements.

$\checkmark$ Intervention phase represents was considered the implementation of the intervention. During this phase, repeated measurements of the dependent variable, using the same measures as assessment checklist and a written record of observations, are obtained to help determine if any change has occurred. This allows determining if the intervention is effective or not. The sessions took place in a natural learning environment, his classroom, at a table, where researcher and participant would sit next to each other. Mobile app (TAC) was installed on a smart device and, given the availability for the purpose of evaluation; the sessions were carried per week, using the TAC. Given that children with autism have a predilection for visual media, this population tends to find the mobile app (TAC) engaging and motivating. 


\section{Results}

Our goal for this study was to see if autistic children could enhance some language concepts through using mobile app (TAC) activities. To assess a subject progress it is necessary to perform measurements. These measurements are acquired through direct observation and should be made prior to and during the intervention with regular periodicity (hours, days, weeks). In this section, we present and discuss the data collected, and what were the results of each phase and of the research as a whole. On the first sessions based on the outcome of the previous session, the first trial was presented without prompt, although it was a step back from the previous session, also showed us that there was an improvement in comparison to the first session of the previous day. The next sessions presented more prompt correctly. On the phase, mobile app (TAC) activities performed both sessions correctly, the word was considered acquired and the intervention phase was complete as shown in Figure .3.

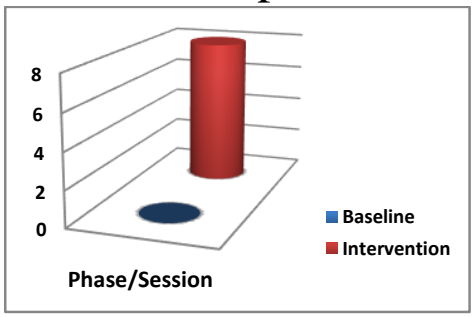

Fig. 3 Phase/Session

Although it was a good result, it can mean that autistic children memorized the answer from the previous session, and that is why we stipulated that there had to be two correct sessions in one day two consider the word as acquired.

The study pointed to several positive functions of the mobile app (TAC) and its impact on improving learning skills of autistic children as shown in Figure .4 


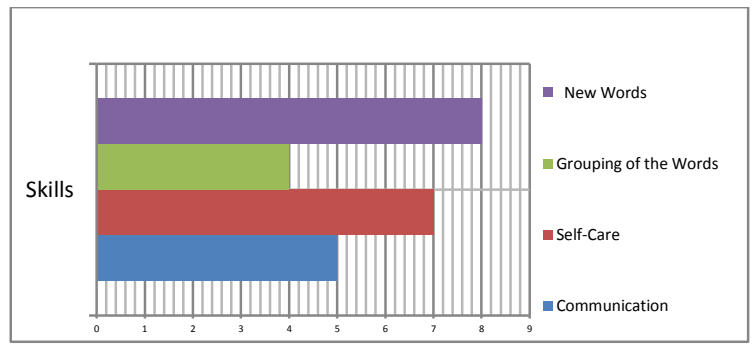

Figure 4 Learning Skills

The study revealed several benefits of the mobile app (TAC) on enhancing language concepts of autistic children as shown in Figure .4

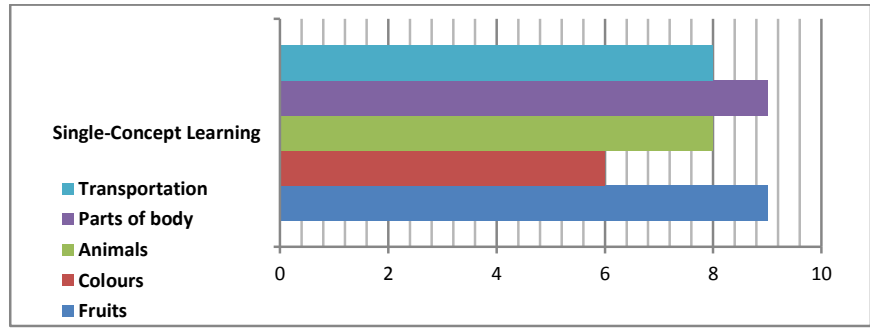

Figure .4 Language Concepts

The results of the study:

1. The study proved the validity of the first hypothesis which states that, There are statistically significant differences between the mean ranks of the children on the the pre/post mobile application in favor of the post application.

2. The study proved the validity of the second hypothesis which states that, There are no statistically significant differences between the mean ranks of the children in the post applications and the follow-up of the mobile application.

The study referred to positive functions of the mobile app (TAC) on language concepts as follows:

- Increased effectiveness of learning new words. 
- Focused on a limited number of words in a week.

- More systematic repetitions than usually done.

- Children considered using the application as a form of a game.

- Children started using newly acquired words, and truly enjoyed it.

- Children prepared pronunciation and an image.

\section{5- Discussion}

Consistent with the research findings involving autistic children, results of this study demonstrated that the suggested mobile app (TAC) is an effective means of improving language concepts for autistic children. The mobile app appeared to have a significant impact. On measuring, there is strong empirical evidence suggesting that mobile app contributes to the development of participants' language concepts. The autistic children who received (TAC) significantly and consistently performed on measures of word development. These results indicate that (TAC) is an effective means of autistic children learning how to pronounce and repeat new words accurately during their learning experience. These findings suggest that the use of the mobile app is effective as a tool and are consistent with the outcomes of the research we conducted previously. Findings showed that (TAC) was effective in enhancing language concepts. This study confirmed what has been demonstrated by others researchers that using mobile apps are an effective means of developing language concepts of 
children with autism. Moreover, the current study proved the effectiveness of the mobile app (TAC) to enhance some language concepts of autistic children.

\section{6- Conclusion}

The research has significant contributions, to advance the active learning of the autistic children. It is to facilitate to enable communication for those children who suffer from Autism. The mobile app was applied to a sample of two autistic children. The result of the study reveals the positive outcome, satisfactory usability and improved words in enhancing some language concepts of autistic children. Efficiency was established using the autistic children mobile app (TAC) which autistic children benefit from the mobile app in enhancing language concepts.

\section{7- Recommendations}

Although we consider this application successful and that it fulfilled our goals. Based on the results of the current study, the following recommendations are stated:

- Mobile Apps should be emphasized in teaching and learning different features of the English language. It includes the use of various activities directed to the autistic children. It makes learning easy for them to absorb knowledge and develop literacy skills. 
- Future research is needed to determine if other programs are as effective as Mobile Apps in helping children with autism's skills. They acquire some of the key foundation skills necessary for their reading success.

- Mobile Apps are also needed to be used with the learning stages. Mobile App is an engaging and developmentally appropriate program that is relevant at all stages.

\section{References}

[1] Fu, F.-L., Su, R.-C., and Yu, S.-C. (2009). 'EGameFlow: A scale to measure learners' enjoyment of e-learning games',Computers \& Education, 52(1), pp. 101-112.[Online]. Available at:

http://www.sciencedirect.com/science/article/pii/S03601315080010 24

2] Bausch, M. E., Ault, M. J., \& Hasselbring, T. S. (2015). Assistive Technology in Schools: Lessons Learned from the National Assistive Technology Research Institute. In Efficacy of Assistive Technology Interventions (pp. 13-50). Emerald Group Publishing Limited.

[3] Sanchez-Gordon, S., \& Luján-Mora, S. (2016). How Could MOOCs Become Accessible? The Case of edX and the Future of 
Inclusive Online Learning. Journal of Universal Computer Science, 22(1), 55-81.

[4] McNaughton D, Light J. (2013). The iPad and mobile technology revolution: Benefits and challenges for individuals who require augmentative and alternative communication. Augmentative and Alternative Communication 2013;29:107-116.

[5] Paparella, T., Goods, K., Freeman, S., \& Kasari, C. (2011). The emergence of nonverbal joint attention and requesting skills in young children with autism. Journal Of Communication Disorders, 44(6), 569-583.

[6] Anderson DK, Lord C, Risi S, DiLavore PS, Shulman C, Thurm A, Welch K, Pickles A. (2007). Patterns of growth in verbal abilities among children with autism spectrum disorder. $J$ Consult Clin Psychol. PAug; 75(4):594-604.

[7] Lofland K. B. (2016). The use of technology in the treatment of autism, in Technology and the Treatment of Children with Autism Spectrum Disorder, ed Cardon T. A., editor. (Cham: Springer International Publishing; ), 27-35.

[8] Hartley C., Allen M. L. (2014b). Intentions vs. resemblance: Understanding pictures in typical development and autism. Cognition 131, 44-59. 10.1016/j.cognition.2013.12.009 
[9] Hartley C., Allen M. L. (2015). Symbolic understanding of pictures in low-functioning children with autism: the effects of iconicity and naming. J. Autism Dev. Disord. 45, 15-30. 10.1007/s10803-013-2007-4 [PubMed] [Cross Ref] [10] Urturi, Z.S., Zorrilla, A.M. \& Zapirain, B.G. (2011). Serious Game based on first aid education for individuals with Autism Spectrum Disorder (ASD) using android mobile devices. Computer Games (CGAMES), 16th International Conference on, pp. 223-7, 2011.

[11] American Psychiatric Association (2013). Diagnostic and statistical manual of mental disorders (5th Edition). Washington, D.C: Author.

[12] Roberts, JM. (2014) Echolalia and language development in children with ASD. Commun Autism 11:55-74.

[13] Hudry .K, Leadbitter K, Temple K, Slonims V, McConachie H, et al. (2010) Preschoolers with autism show greater impairment in receptive compared with expressive language abilities, Int. $J$ Lang Commun Disord 45: 681-690.

[14] Wiig, E. H. (2004). Wiig assessment of basic concepts ®. Greenville, SC: Super Duper ${ }^{\circledR}$ Publications. 